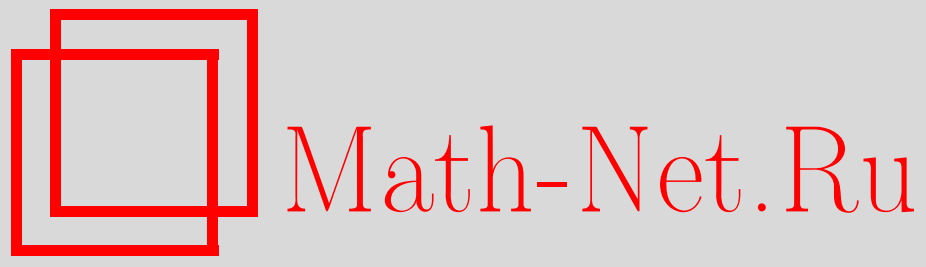

И. Г. Царьков, Локальное сглаживание равномерно гладких отображений, Функи. анализ и его прил., 2006, том 40, выпуск 3, 44-52

DOI: https://doi.org/10.4213/faa742

Использование Общероссийского математического портала Math-Net.Ru подразумевает, что вы прочитали и согласны с пользовательским соглашением

http://www. mathnet.ru/rus/agreement

Параметры загрузки:

IP : 54.162 .127 .20

26 апреля 2023 г., 16:05:40

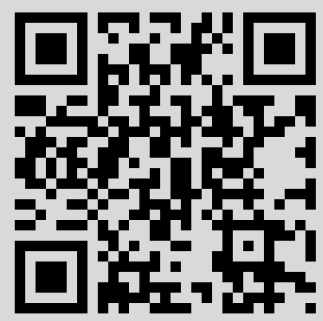


Функииональный анализ и его приложения

2006, т. 40, вып. 3, с. 44-52

УДК 517.17

\title{
Локальное сглаживание равномерно гладких отображений
}

\author{
(с) 2006. И. Г. ЦАРьков
}

\section{$\S 1$. Введение}

В работе решается задача равномерного приближения равномерно непрерывных (гладких) отображений отображениями, имеющими по возможности наибольшую локальную и равномерную гладкость (см теоремы 1-3).

Одной из первых аппроксимативных теорем в бесконечномерных пространствах в случае равномерной аппроксимации была теорема Бонича и Фремптона (см. [1]), которая устанавливает тесную связь между гладкостью банахова пространства $X$ и аппроксимативными свойствами гладких функций на $X$. Под гладкостью пространства $X$ эти авторы понимают такое наибольшее число $r$ (может быть, $\infty)$, для которого существует ненулевая финитная функция класса $C^{r}(X, \mathbb{R})$. Они доказали, что если $X$ - сепарабельное банахово пространство гладкости $r$ (в этом смысле), то для любых $\varepsilon>0$, банахова пространства $Y$, открытого множества $U \subset X$ и отображения $f \in C(U, Y)$ существует сглаживающее отображение $g \in C^{r}(U, Y)$, такое, что $\|f-g\|<\varepsilon$. Имеются варианты этой теоремы и для аналитических функций (см. [2]). Отметим, что сглаживающие отображения здесь не являются равномерно гладкими. Валентайном [3] была получена теорема о возможности продолжения гильбертовозначного липшицева отображения с произвольного подмножества гильбертова пространства на все это пространство с сохранением константы Липшица. Отсюда легко выводится, что всякое равномерно непрерывное гильбертовозначное отображение, заданное на произвольном подмножестве гильбертова пространства, можно равномерно приблизить равномерно липшицевым отображением. Сглаживанием равномерно непрерывных действительных функций, заданных на шарах в гильбертовых пространствах и пространствах $L_{p}$, многочленами и представителями некоторых классов равномерно гладких функций занимались также Немировский и Семенов [4]-[7]). Дальнейшее развитие эта тематика получила в работах автора [8]-[17]. Упомянем также монографию Беньямини и Линденштраусса [18], в которой, в частности, приводится обзор по тематике приближения и продолжения равномерно непрерывных отображений.

В работах автора [8], [17] изучались задачи равномерного приближения равномерно непрерывного отображения в пространствах $L_{p}$ функциями, имеющими максимально возможную локальную и равномерную гладкость. В настоящей работе продолжается исследование этих вопросов в более общих пространствах. В частности, для равномерно гладких отображений, определенных на простран-

* Работа выполнена при финансовой поддержке Российского фонда фундаментальных исследований (грант 06-01-00160). 
стве $X$ с безусловным конечномерным шаудеровским разложением, строится равномерное приближение отображениями максимально возможной локальной гладкости (той же, что и у пространства $X$ ) с сохранением равномерной гладкости.

ОПРеДЕЛЕНИЕ 1. Пусть $X, Y$ - банаховы пространства и $M \subset X$. Скажем, что отображение $f: M \rightarrow Y$ принадлежст классу $H^{\alpha}(M)=H^{\alpha}(M, Y)(\alpha>0)$, если оно лежит в $D^{\beta}(M)$ и все его производные до порядка $\beta$ включительно ограничены на $M$, a $f^{(\beta)}$ принадлежит классу Гёльдера порядка $\alpha-\beta$ на множестве $M$, где $\beta$ - наибольшее целое число, строго меньшее $\alpha$. Если условия Гёльдера рассматривать локально, то получатся классы $H_{\mathrm{loc}}^{\alpha}(M)=H_{\mathrm{loc}}^{\alpha}(M, Y)$. При этом под классом $H^{\infty}(M)=H^{\infty}(M, Y)$ мы будем подразумевать класс всех бесконечно дифференцируемых отображений $f: M \rightarrow Y$, производные которых ограничены на множестве $M$, а под классом $H_{\mathrm{loc}}^{\infty}(M)=H_{\mathrm{loc}}^{\infty}(M, Y)-$ класс всех бесконечно дифференцируемых отображений $f: M \rightarrow Y$. Будем называть отображение $f$ из $H^{\alpha}(M, Y)$ отображением гладкости $\alpha$, a $f$ из $H_{\mathrm{loc}}^{\alpha}(M, Y)$ отображением локальной гладкости $\alpha$. Здесь, как обычно, мы считаем, что $f^{(0)} \stackrel{\text { def }}{=} f$.

ОПреДЕЛЕниЕ 2. Через ( $\left.\mathscr{H}^{\alpha}\right)$ обозначим класс всех действительных банаховых пространств $X$, таких, что норма на $X$ принадлежит классу $H^{\alpha}(X \backslash B, \mathbb{R})$, где $B=\{x \in X \mid\|x\| \leqslant 1\}$ - единичный шар в этом пространстве. Такие пространства будем называть $\alpha$-гладкими.

В настоящей работе получен следующий результат.

Tеорема 1. Пусть $X \in\left(\mathscr{H}^{\alpha}\right), \alpha \geqslant 1$, - банахово пространство, обладающее набором конечномерных подпространств $E=\left\{L_{m}^{\prime}\right\}_{m \in \mathbb{N}}$, представляющих собой его безусловное шаудеровское разложсние, и $r \in(0, \alpha]$. Тогда для любого $\varepsilon>0$ и произвольных банахова пространства $Y$ и отображения $f \in H^{r}(X, Y)$ существует локально $\alpha$-гладкое отображсение $\varphi \in H^{r}(X, Y)$, maкое, что $\|f-\varphi\| \leqslant \varepsilon$.

На основе этой теоремы и прежних результатов автора доказываются также следующие теоремы:

Теорема 2. Пусть $X \in\left(\mathscr{H}^{\alpha}\right), \alpha \geqslant 2$, - банахово пространство, обладающее набором конечномерных подпространств $E=\left\{L_{m}^{\prime}\right\}_{m \in \mathbb{N}}$, представляющих собой его безусловное шаудеровское разложсение. Тогда существует число $C=C(X)>0$, такое, что для любого равномерно непрерывного отображения $f: X \rightarrow l_{2}$ и произвольного $\varepsilon>0$ найдется локально $\alpha$-гладкое $и C \frac{\omega(f, \varepsilon)}{\varepsilon}-л и п-$ шищево отображсение $\varphi: X \rightarrow l_{2}$, для которого $\|f-\varphi\| \leqslant C \omega(f, \varepsilon)$.

Здесь через $l_{2}$ обозначено пространство действительных последовательностей $x=\left(x_{1}, x_{2}, \ldots\right)$ с нормой $\|x\|_{2} \stackrel{\text { def }}{=}\left(\sum_{i=1}^{\infty}\left|x_{i}\right|^{2}\right)^{1 / 2}$.

Теорема 3. Пусть $f: l_{2} \rightarrow \mathbb{R}$ - равномерно непрерывная функиия. Тогда существует абсолютная константа $C>0$, такая, что для любого $\varepsilon>0$

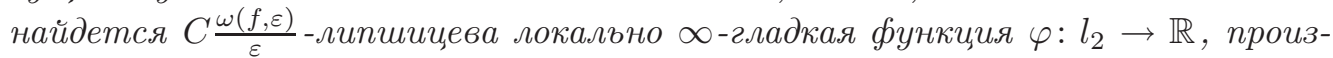
водная которой является $C \frac{\omega(f, \varepsilon)}{\varepsilon^{2}}$-липшищевым отображсением и для которой $\|f-\varphi\| \leqslant C \omega(f, \varepsilon)$. 


\section{§2. Основные конструкции}

Пусть $X-$ действительное банахово пространство, а $E=\left\{L_{m}^{\prime}\right\}_{m \in \mathbb{N}}$ - набор его подпространств, представляющий собой шаудеровское разложение пространства $X$, т. е. каждый элемент $x \in X$ однозначно представляется в виде суммы $\sum_{m \in \mathbb{N}} x_{m}$, где $m$-й член $x_{m}$ разложения принадлежит $L_{m}^{\prime}$ (см. [19]). При этом оператор $T_{m}$, ставящий в соответствие элементу $x m$-й член его разложения, будет непрерывным проектором на $L_{m}^{\prime}$. Такое разложение называется безусловным, если существует константа $C=C(E)>0$ (константа безусловности), такая, что для любых $x=\sum_{m \in \mathbb{N}} x_{m} \in X, n \in \mathbb{N}$, всех наборов чисел $\alpha_{i} \in \mathbb{R}$ и $\kappa_{i} \in\{1,-1\}(i=1, \ldots, n)$ выполняются неравенства

$$
C^{-1}\left\|\sum_{i=1}^{n} \kappa_{i} \alpha_{i} x_{n}\right\| \leqslant\left\|\sum_{i=1}^{n} \alpha_{i} x_{n}\right\| \leqslant C\left\|\sum_{i=1}^{n} \kappa_{i} \alpha_{i} x_{n}\right\| .
$$

При этом линейное многообразие $\mathscr{L}=\bigcup_{m=1}^{\infty} L_{m}$, где $L_{m} \stackrel{\text { def }}{=} L_{1}^{\prime} \oplus \cdots \oplus L_{m}^{\prime}$, всюду плотно в $X$, а $P_{m}=\sum_{i=1}^{m} T_{m}$ является проектором пространства $X$ на $L_{m}$ и без потери общности можно считать, что его норма, а также норма оператора $Q_{m}=\mathrm{Id}-P_{m}$ ограничены той же константой $C$.

Через $\varrho(x, G)$ обозначим расстояние от точки $x \in X$ до некоторого множества $G \subset X$, т. е. величину $\inf \{\|x-y\| \mid y \in G\}$. Отметим простейшее свойство проекторов $P_{i}: P_{m}=P_{m+1} \circ P_{m}=P_{m}^{2}$ для всех $m \in \mathbb{N}$. Отметим также, что последовательность $\left\{\varrho\left(x, L_{k}\right)\right\}$ монотонно убывает по $k$. Через $B(x, r)$ обозначим шар с центром $x$ радиуса $r$ в пространстве $X$, т. е. множество $\{y \in X \mid\|x-y\| \leqslant r\}$. В силу того, что линейное многообразие $\mathscr{L}$ всюду плотно в $X$, для произвольного элемента $x \in X$ выполняется равенство $\lim _{k \rightarrow \infty} P_{k}(x)=x$. Пусть $\Delta \in(0,1 / 3)$, и пусть $\varphi^{\Delta}: \mathbb{R}_{+} \rightarrow \mathbb{R}_{+}-$монотонно возрастающая бесконечно дифференцируемая функция, являющаяся $(1-3 \Delta)^{-1}$-липшицевой и такая, что $\varphi^{\Delta} \equiv 0$ на $[0, \Delta], \varphi^{\Delta} \equiv 1$ на $[1,+\infty)$. Для произвольных $\varepsilon>0$ и $\delta \in(0, \varepsilon / 3)$ введем бесконечно дифференцируемую монотонно возрастающую функцию $\varphi(t)=\varphi_{\delta}(t) \stackrel{\text { def }}{=} \varphi^{\Delta}(t / \varepsilon): \mathbb{R}_{+} \rightarrow \mathbb{R}_{+}$, где $\Delta=\delta / \varepsilon$. Функция $\varphi$ является $C_{\delta}$-липшицевой, где $C_{\delta}=1 /(\varepsilon-3 \delta)$, и $\varphi \equiv 0$ на $[0, \delta], \varphi \equiv 1$ на $[\varepsilon,+\infty)$. В дальнейшем будем считать, что $\delta=\varepsilon / 9$. В этом случае равномерные нормы $k$-х производных функции $\varphi$ имеют порядок $O\left(\varepsilon^{-k}\right)$.

Для всех $x \in X$ и $n \in \mathbb{N}$ положим $\theta_{n}(x)=\varphi \circ \varrho_{n}(x)$, где $\varrho_{n}(x)=\left\|x-P_{n} x\right\|=$ $\left\|Q_{n}(x)\right\|$, и $e_{n}(x)=P_{n}(x)-P_{n+1}(x)=-T_{n+1}(x)=-x_{n+1}$. Отметим, что для пространства $X \in\left(\mathscr{H}^{\alpha}\right), \alpha>0$, и наибольшего целого числа $\beta$, строго меньшего $\alpha$ (если $\alpha=\infty$, то полагаем $\beta=\infty$ ), существует набор, состоящий из положительных чисел $K_{n}, n=0, \ldots, \beta$, и $K_{\alpha}$, таких, что $\left\|\theta_{k}^{(n)}\right\| \leqslant K_{n} \varepsilon^{-n}(n=0, \ldots, \beta)$ и $\left\|\theta_{k}^{(\beta)}(x)-\theta_{k}^{(\beta)}(y)\right\| \leqslant K_{\alpha} \varepsilon^{-\alpha}\|x-y\|^{\gamma}(\gamma=\alpha-\beta)$ для любого $k \in \mathbb{N}$ и для всех $x, y \in X$.

Рассмотрим отображение $F(x)=x+\sum_{k=1}^{\infty}\left(1-\theta_{k}(x)\right) e_{k}(x)(x \in X)$. Для каждого элемента $x \in X$ введем числа $m=m(x)=\min \left\{k \in \mathbb{N} \mid \varrho\left(x, L_{k}\right)<\delta / C\right\}$ и

$$
l=l(x)= \begin{cases}\max \left\{k \in \mathbb{N} \mid \varrho\left(x, L_{k}\right)>\varepsilon\right\}, & \text { если } \varrho\left(x, L_{1}\right)>\varepsilon, \\ 0, & \text { если } \varrho\left(x, L_{1}\right) \leqslant \varepsilon .\end{cases}
$$


Нетрудно проверить, что $l<m$. В силу определения этих чисел $\varrho_{n}(x)<\delta$ для любого $n \geqslant m+1$ и $\varrho_{n}(x) \geqslant \varepsilon$ для любого $n \leqslant l$, а следовательно, $F(x)=$ $x+\sum_{k=m+1}^{\infty} e_{k}(x)+\sum_{k=l+1}^{m}\left(1-\theta_{k}(x)\right) e_{k}(x)=P_{m+1}(x)+\sum_{k=l+1}^{m}\left(1-\theta_{k}(x)\right) e_{k}(x)$.

\section{§3. Вспомогательные утверждения}

Следующую лемму можно найти в работах [19], [20].

ЛЕмма 1. Для произвольного элемента $x \in X$ и любых чисел $\alpha_{k} \in \mathbb{R}(k=$ $l+1, \ldots, m)$

$$
\left\|\sum_{k=l+1}^{m} \alpha_{k} e_{k}(x)\right\| \leqslant C\|x\| \max _{k=l+1, \ldots, m}\left|\alpha_{k}\right| .
$$

Докажем, что образ $F(x)$ каждого элемента $x \in X$ принадлежит $B\left(x, \varepsilon_{0}\right)$, где $\varepsilon_{0}=\left(C+C^{2}\right) \varepsilon$. Для этого сначала заметим, что $\left\|x-P_{k}(x)\right\| \leqslant C \varrho\left(x, L_{k}\right) \leqslant C \varepsilon$ для всех $k \geqslant l+1$. Поэтому в силу леммы 1 , учитывая, что $e_{k}(x)=e_{k}\left(x-P_{l+1}(x)\right)$ для всех $k \geqslant l+1$, получим оценку $\|F(x)-x\| \leqslant\left\|P_{m+1}(x)-x\right\|+\| \sum_{k=l+1}^{m}(1-$ $\left.\theta_{k}(x)\right) e_{k}(x)\|\leqslant C \varepsilon+\| \sum_{k=l+1}^{m}\left(1-\theta_{k}(x)\right) e_{k}\left(x-P_{l+1}(x)\right)\|\leqslant C \varepsilon+C\| x-P_{l+1}(x) \| \leqslant$ $C \varepsilon+C^{2} \varepsilon=\varepsilon_{0}$. Следовательно, $F(x) \in B\left(x, \varepsilon_{0}\right)$.

Докажем, что при $\delta=\varepsilon / 9$ отображение $F$ является липшицевым с константой Липшица, равной $D=3 C^{2}+2 C$. Для этого достаточно доказать, что в некоторой окрестности произвольного элемента $x \in X$ это отображение $D$-липшицево. Возьмем такую малую окрестность элемента $x$, чтобы $l(x) \leqslant l(y)$ и $m(x) \geqslant m(y)$ для любого элемента $y$ из этой окрестности. Тогда $F(y)=$ $P_{m+1}(y)+\sum_{k=l+1}^{m}\left(1-\theta_{k}(y)\right) e_{k}(y)$, где $m=m(x), l=l(x)$, и

$$
\begin{aligned}
\|F(x)-F(y)\| \leqslant & \left\|P_{m+1}(x-y)\right\|+\left\|\sum_{k=l+1}^{m}\left(\theta_{k}(y)-\theta_{k}(x)\right) e_{k}(x)\right\| \\
& +\left\|\sum_{k=l+1}^{m}\left(1-\theta_{k}(y)\right)\left(e_{k}(x)-e_{k}(y)\right)\right\| \\
\leqslant & C\|x-y\|+\left\|\sum_{k=l+1}^{m}\left(\theta_{k}(y)-\theta_{k}(x)\right) e_{k}\left(x-P_{l+1}(x)\right)\right\| \\
& +\left\|\sum_{k=l+1}^{m}\left(1-\theta_{k}(y)\right)\left(e_{k}(x-y)\right)\right\| \\
\leqslant & C\|x-y\|+C C_{\delta}\|x-y\|\left\|x-P_{l+1}(x)\right\|+C\|x-y\| \\
\leqslant & \|x-y\|\left(C+C^{2} C_{\delta} \varepsilon+C\right) \leqslant\|x-y\|\left(2 C+C^{2} \frac{\varepsilon}{\varepsilon-3 \delta}\right) .
\end{aligned}
$$

Отсюда получим, что

$$
\|F(x)-F(y)\| \leqslant\left(2 C+3 C^{2}\right)\|x-y\|=D\|x-y\|,
$$

т. е. $F$ является липшицевым отображением с константой Липшица, равной $D$. Кроме того, для $X \in\left(\mathscr{H}^{\alpha}\right), \alpha>0, p \in \mathbb{Z}_{+}, p<\alpha$, и $\gamma=\min \{1, \alpha-p\}$ 
выполняются неравенства

$$
\begin{aligned}
\| F^{(p)}( & x)\left[h_{1}, \ldots, h_{p}\right]-F^{(p)}(y)\left[h_{1}, \ldots, h_{p}\right] \| \\
= & \| \sum_{k=l+1}^{m} \theta_{k}^{(p)}(y)\left[h_{1}, \ldots, h_{p}\right] e_{k}(y)-\theta_{k}^{(p)}(x)\left[h_{1}, \ldots, h_{p}\right] e_{k}(x) \\
& +\sum_{k=l+1}^{m} \sum_{i=1}^{p}\left(\theta_{k}^{(p-1)}(y)-\theta_{k}^{(p-1)}(x)\right)\left[h_{1}, \ldots, h_{i-1}, h_{i+1}, \ldots, h_{p}\right] e_{k}\left(h_{i}\right) \| \\
\leqslant & \left\|\sum_{k=l+1}^{m}\left(\theta_{k}^{(p)}(y)\left[h_{1}, \ldots, h_{p}\right]-\theta_{k}^{(p)}(x)\left[h_{1}, \ldots, h_{p}\right]\right) e_{k}(x)\right\| \\
& +\left\|\sum_{k=l+1}^{m}\left(-\theta_{k}^{(p)}(y)\left[h_{1}, \ldots, h_{p}\right]\right) e_{k}(x-y)\right\| \\
& +\sum_{i=1}^{p}\left\|\sum_{k=l+1}^{m}\left(\theta_{k}^{(p-1)}(y)-\theta_{k}^{(p-1)}(x)\right)\left[h_{1}, \ldots, h_{i-1}, h_{i+1}, \ldots, h_{p}\right] e_{k}\left(h_{i}\right)\right\| \\
\leqslant & \left\|\sum_{k=l+1}^{m}\left(\theta_{k}^{(p)}(y)\left[h_{1}, \ldots, h_{p}\right]-\theta_{k}^{(p)}(x)\left[h_{1}, \ldots, h_{p}\right]\right) e_{k}\left(x-P_{l+1} x\right)\right\| \\
& +C K_{p} \varepsilon^{-p}\|x-y\|+p C K_{p} \varepsilon^{-p}\|y-x\| \\
\leqslant & K_{p, \gamma} \varepsilon^{-p-\gamma}\|y-x\|_{p, \gamma} \varepsilon^{-p-\gamma+1}\left\|y-P_{l+1} x\right\|+C K_{p}(p+1) \varepsilon^{-p}\|x-y\|
\end{aligned}
$$

где $K_{p, \gamma} \in\left\{K_{\alpha}, K_{p+1}\right\}$, a $h_{1}, \ldots, h_{p}$ - произвольные единичные векторы. Отсюда следует, что $F \in H^{\alpha}(X, X)$.

Лемма 2. Пусть $X \in\left(\mathscr{H}^{\alpha}\right)$. Для каждого $\varepsilon_{0}>0$ введенное выше (для линейного многообразия $\left.\mathscr{L}=\bigcup_{m=1}^{\infty} L_{m} u \varepsilon=\varepsilon_{0}\left(C^{2}+C\right)^{-1}\right)$ D-липшицево отображсение $F: X \rightarrow \mathscr{L}$ удовлетворяет следующим соотношениям:

1) $\|x-F(x)\| \leqslant \varepsilon_{0}$ для всех $x \in X$;

2) для каждого элемента $x \in X$ существует номер $n=n(x)$, такой, что $F\left(B\left(x, \frac{\varepsilon_{0}}{36 C\left(C+C^{2}\right)}\right)\right) \subset L_{n}$.

Доказательство. Положим $\varepsilon=\varepsilon_{0}\left(C^{2}+C\right)^{-1}$. Выберем $K=K(x) \in \mathbb{N}$ так, чтобы $\varrho\left(x, L_{K}\right)<\frac{\varepsilon_{0}}{36 C\left(C+C^{2}\right)}$. Так как $\varrho\left(y, L_{K}\right) \leqslant \varrho\left(x, L_{K}\right)+\|x-y\|<$ $\frac{\varepsilon_{0}}{18 C\left(C+C^{2}\right)}=\frac{\varepsilon}{18 C} \leqslant \frac{\delta}{C}$ для всех $y \in B\left(x, \frac{\varepsilon_{0}}{36 C\left(C+C^{2}\right)}\right)$, то $\left\|y-P_{K}(y)\right\| \leqslant \delta$ и $F(y)=$ $P_{K+1}(y)+\sum_{k=1}^{K}\left(1-\theta_{k}(y)\right) e_{k}(y) \in L_{K+1}$. Следовательно, $F\left(B\left(x, \frac{\varepsilon_{0}}{36 C\left(C+C^{2}\right)}\right)\right) \subset$ $\mathscr{L}_{K+1}$. Остальные свойства доказаны ранее.

\section{§4. Доказательства теорем}

ДокаЗАТЕЛЬСтво теоремы 1. Рассмотрим сначала случай, когда $r \in(0,1]$. Тогда существует константа $K>0$, такая, что $\left\|f\left(x_{1}\right)-f\left(x_{2}\right)\right\| \leqslant K\left\|x_{1}-x_{2}\right\|^{r}$ для всех $x_{1}, x_{2} \in X$, т. е. $f$ - гёльдерово отображение с показателем $r$ и константой $K$. Пусть $\varepsilon_{1}=\left(\frac{\varepsilon}{2 K+1}\right)^{1 / r}$. По лемме 2 существует $\alpha$-гладкое $D$-липшицево отображение $F: X \rightarrow \mathscr{L}=\bigcup_{m=1}^{\infty} L_{m}\left(L_{m} \stackrel{\text { def }}{=} L_{1}^{\prime} \oplus \cdots \oplus L_{m}^{\prime}\right)$, такое, что 
$\|x-F(x)\| \leqslant \varepsilon_{1}$ для всех $x \in X$ и найдется такое число $m=m(x) \in \mathbb{N}$, что $F\left(B\left(x, \frac{\varepsilon_{1}}{36 C\left(C+C^{2}\right)}\right)\right) \subset L_{m}(C-$ константа из леммы 2$)$.

Идея доказательства состоит в следующем. Мы построим отображение $f_{\infty}$ : $X \rightarrow Y$, бесконечно дифференцируемое по линейному многообразию $\mathscr{L}$ и приближающее отображение $f$. Такое отображение строится путем последовательных сверток отображения $f$ с бесконечно гладкими действительными функциями по направлениям, линейная оболочка которых равна $\mathscr{L}$. Искомое отображение $\varphi$ представляет собой композицию отображения $f_{\infty}$ и отображения $F$, образ которого локально содержится в конечномерном подпространстве из $\mathscr{L}$.

Пусть $\left\{v_{j}\right\}_{j \in \mathbb{N}}$ - набор единичных векторов из $\mathscr{L}$, удовлетворяющих следующему условию: для каждого $m \in \mathbb{N}$ найдется $n_{m} \in \mathbb{N}$, такое, что линейная оболочка векторов $\left\{v_{j}\right\}_{j=1}^{n_{m}}$ содержит подпространство $L_{m}$. Рассмотрим неотрицательную финитную бесконечно дифференцируемую функцию $\tau: \mathbb{R} \rightarrow \mathbb{R}$, носитель которой содержится в отрезке $[-1,1]$, такую, что $\int_{\mathbb{R}} \tau(t) d t=1$. Для произвольных $a>0$ и $j \in \mathbb{N}$ определим оператор $R_{j, a}: C(X, Y) \rightarrow C(X, Y)$, положив $g_{j, a}=R_{j, a}(g(x))=\int_{\mathbb{R}} g\left(x+v_{j} t\right) \tau_{a}(t) d t=g \star \tau_{a}$, где $\tau_{a}(t)=\frac{1}{a} \tau\left(\frac{t}{a}\right)$. Тогда

$$
\begin{aligned}
\frac{\partial^{k} g_{j, a}}{\partial v_{j}^{k}}(x) & =\left.\frac{\partial^{k}}{\partial u^{k}} R_{j, a}\left(g\left(x+u v_{j}\right)\right)\right|_{u=0}=\left.\frac{\partial^{k}}{\partial u^{k}} \int_{\mathbb{R}} g\left(x+v_{j}(u+t)\right) \tau_{a}(t) d t\right|_{u=0} \\
& =\left.\frac{\partial^{k}}{\partial u^{k}} \int_{\mathbb{R}} g\left(x+v_{j} v\right) \tau_{a}(v-u) d v\right|_{u=0} \\
& =\left.\int_{\mathbb{R}} g\left(x+v_{j} v\right) \frac{\partial^{k}}{\partial u^{k}} \tau_{a}(v-u)\right|_{u=0} d v \in C(X, Y) .
\end{aligned}
$$

Аналогично,

$\frac{\partial^{k}}{\partial v_{1}^{k_{1}} \cdots \partial v_{n}^{k_{n}}} R_{j_{1}, a_{1}} \circ \cdots \circ R_{j_{n}, a_{n}}(g)=(-1)^{k} g \star \frac{\partial^{k_{1}}}{\partial v_{1}^{k_{1}}} \tau_{a_{1}} \star \cdots \star \frac{\partial^{k_{n}}}{\partial v_{n}^{k_{n}}} \tau_{a_{n}} \in C(X, Y)$,

и, более того, верно следующее утверждение.

ЗАмЕчАниЕ 1 . Если отображение $g$ ограничено на шаре $B\left(x, \sum_{i=1}^{n} a_{i}\right)$, то норма производной из левой части формулы (2) ограничена константой, которая зависит только от чисел $a_{1}, \ldots, a_{n}$, числа $k=k_{1}+\cdots+k_{n}$ и величины $\sup _{y \in B\left(x, \sum_{i=1}^{n} a_{i}\right)}\|g(y)\|$. Кроме того, для всех пар $(j, a)$ выполняется неравенство $\left\|g_{j, a}\right\| \leqslant \sup _{y \in B(x, a)}\|g\|$.

Далее, для любой точки $x \in X$

$$
\begin{aligned}
\left\|g(x)-g_{j, a}(x)\right\| & =\left\|\int_{\mathbb{R}} g(x) \tau_{a}(t) d t-\int_{\mathbb{R}} g\left(x+v_{j} t\right) \tau_{a}(t) d t\right\| \\
& =\left\|\int_{\mathbb{R}}\left(g(x)-g\left(x+v_{j} t\right)\right) \tau_{a}(t) d t\right\| \\
& =\left\|\int_{-a}^{a}\left(g(x)-g\left(x+v_{j} t\right)\right) \tau_{a}(t) d t\right\| \leqslant \omega(g, a) \int_{-a}^{a} \tau_{a}(t) d t \leqslant \omega(g, a),
\end{aligned}
$$


т. е. норма разности значений $g(x)$ и $g_{j, a}(x)$ оценивается через модуль непрерывности отображения $g$ с шагом $a$. Для произвольных $x_{1}, x_{2} \in X$ верна оценка

$$
\left\|g_{j, a}\left(x_{1}\right)-g_{j, a}\left(x_{2}\right)\right\|=\left\|\int_{\mathbb{R}}\left(g\left(x_{1}+v_{j} t\right)-g\left(x_{2}+v_{j} t\right)\right) \tau_{a}(t) d t\right\| \leqslant \omega\left(g,\left\|x_{1}-x_{2}\right\|\right) .
$$

Следовательно, $\omega\left(g_{j, a}, t\right) \leqslant \omega(g, t)$ для всех $t \geqslant 0$.

Положим $a_{n}=\left(\frac{\varepsilon}{2(K+1)} 2^{-n}\right)^{1 / r}(n \in \mathbb{N}), f_{0}=f, f_{n}=R_{n, a_{n}} \circ \cdots \circ R_{1, a_{1}}(f)$. Тогда $\omega\left(f_{n}, t\right) \leqslant \omega(f, t)$ для всех $n \in \mathbb{N}(t \geqslant 0)$ и

$\left\|f_{n}-f_{n+1}\right\| \leqslant \omega\left(f_{n}, a_{n+1}\right) \leqslant \omega\left(f_{n-1}, a_{n+1}\right) \leqslant \cdots \leqslant \omega\left(f, a_{n+1}\right) \leqslant K\left(a_{n+1}\right)^{r}<\frac{\varepsilon}{2^{(n+2)}}$.

Следовательно, существует $\lim _{n \rightarrow \infty} f_{n} \stackrel{\text { def }}{=} f_{\infty}$ и отображение $f_{\infty}$ является гёльдеровым с показателем $r$ и константой $K$. В силу замечания 1 для всех $n, m \in \mathbb{N}$, $n \geqslant m$, величина $\left\|\frac{\partial^{k}}{\partial v_{1}^{k_{1}} \ldots \partial v_{m}^{k_{m}}} f_{n}(x)\right\|$ ограничена константой, зависящей только
от $m, k$ и от

$$
A_{m} \stackrel{\text { def }}{=} \sup _{y \in B(x, \varepsilon / 2)}\left\|\frac{\partial^{k}}{\partial v_{1}^{k_{1}} \cdots \partial v_{m}^{k_{m}}} f_{m}(y)\right\| .
$$

Учитывая, что $A_{m}$ в силу замечания 1 зависит только от $m$ и $\sup _{y \in B(x, \varepsilon)}\|f(y)\|$, мы получаем, что $f_{\infty}$ бесконечно дифференцируемо по направлениям из линейного многообразия $\mathscr{L}$. Кроме того, для всех $n \in \mathbb{N}$

$$
\left\|f-f_{n}\right\| \leqslant \sum_{i=1}^{n}\left\|f_{i}-f_{i-1}\right\|<\sum_{i=1}^{n} \frac{\varepsilon}{2^{i+1}}<\frac{\varepsilon}{2} .
$$

Поэтому $\left\|f-f_{\infty}\right\| \leqslant \varepsilon / 2$. Положим $\varphi=f_{\infty} \circ F$, где $F$ - отображение, рассматривавшееся в лемме 2. Для всех точек $x \in X$ верна оценка $\|f(x)-\varphi(x)\| \leqslant$ $\|f(x)-f(F(x))\|+\left\|f(F(x))-f_{\infty}(F(x))\right\| \leqslant K\|x-F(x)\|^{r}+\varepsilon / 2 \leqslant K \varepsilon_{1}+\varepsilon / 2<\varepsilon$.

Рассмотрим теперь случай, когда $r \in(1, \alpha]$. Пусть $Y_{0}=Y$, а $Y_{p}$ - пространство линейных непрерывных операторов из $X$ в $Y_{p-1}(p \in \mathbb{N})$. Положим $f_{0, p}=d^{p} f / d x^{p}: X \rightarrow Y_{p}, f_{n, p}=R_{n, a_{n}} \circ \cdots \circ R_{1, a_{1}}\left(f^{(p)}\right)$. В силу свойств свертки $f_{n, p}=f_{n}^{(p)}$. Так же, как и выше, доказывается, что последовательность $\left\{f_{n, p}=f_{n}^{(p)}\right\}$ фундаментальна и сходится к $f_{\infty, p}=f_{\infty}^{(p)}$. При этом $f_{\infty, p}$ (т. е. $\left.f_{\infty}^{(p)}\right)$ локально $(\alpha-p)$-гладко и гёльдерово с показателем $\beta=\min \{1, r-p\}$. Таким образом, индукцией по $p$ мы получим, что $f_{\infty}$ локально $\alpha$-гладко и принадлежит классу $H^{r}(X, Y)$. Нетрудно проверить, что $\varphi \in H^{r}(X, Y)$, и так как для каждого $x \in X$ найдется число $m=m(x) \in \mathbb{N}$, такое, что $F\left(B\left(x, \frac{\varepsilon_{1}}{36 C\left(C+C^{2}\right)}\right)\right) \subset L_{m}$, a $\left.f_{\infty}\right|_{L_{m}} \in C^{\infty}\left(L_{m}\right)$, то $\varphi$ локально $\alpha$-гладко. Теорема доказана.

ЗАмЕчАниЕ 2. Если в условиях предыдущей теоремы отображение $f$ является $C$-липшицевым, то локально $\alpha$-гладкое отображение $\varphi$ будет $D C$-липшицевым.

ЗАмечАниЕ 3. Если в условиях предыдущей теоремы $f$ является $C$-липшицевым отображением, а его производная - $C^{\prime}$-липшицевой и $\alpha \geqslant 2$, то в силу оценки (1) для $F^{\prime}$ и $F^{\prime \prime}$ найдется абсолютная константа $G>0$, такая, что локально $\alpha$-гладкое отображение $\varphi$ будет иметь $G C^{\prime \prime}$-липшицеву производную, где $C^{\prime \prime}=\max \left\{C / \varepsilon, C^{\prime}\right\}$. 
ДокАЗАТЕЛЬСтво теоремы 2. В [17] было показано, что существует такая константа $K=K(X)>0$, что для любого непустого подмножества $M \subset X$ и произвольных числа $R>0$ и $R$-липшицева отображения $g: M \rightarrow l_{2}$ существует его продолжение $-K R$-липшицево отображение $\hat{g}: X \rightarrow l_{2}$. Возьмем в качестве множества $M \subset X$ максимальное по вложению множество $\varepsilon$-различимых точек, т. е. такое множество, что для произвольных различных точек $x, y \in M$ и любой точки $t \in X$ выполняются неравенства $\|x-y\|_{X} \geqslant \varepsilon, \varrho(t, M)<\varepsilon$. Тогда отображение $g=\left.f\right|_{M}$ является $2 \omega(f, \varepsilon) / \varepsilon$-липшицевым. Действительно, для произвольных различных точек $x, y \in M$ имеем $\|f(x)-f(y)\|_{2} \leqslant$ $\omega\left(f,\|x-y\|_{X}\right) \leqslant\left(\frac{\|x-y\|_{X}}{\varepsilon}+1\right) \omega(f, \varepsilon) \leqslant \frac{2\|x-y\|_{X}}{\varepsilon} \omega(f, \varepsilon)$. Продолжим это отображение до $2 K \frac{\omega(f, \varepsilon)}{\varepsilon}$-липшицева отображения $\hat{g}: X \rightarrow l_{2}$. Для произвольной точки $t \in X$ найдется такая точка $x \in M$, что $\|x-t\|<\varepsilon$. Отсюда следует, что $\|f(t)-\hat{g}(t)\|_{2} \leqslant\|f(x)-f(t)\|_{2}+\|\hat{g}(x)-f(x)\|_{2}+\|\hat{g}(t)-\hat{g}(x)\|_{2}=$ $\|f(x)-f(t)\|_{2}+\|\hat{g}(t)-\hat{g}(x)\|_{2} \leqslant \omega(f, \varepsilon)+2 K \frac{\omega(f, \varepsilon)}{\varepsilon}\|x-t\|_{X} \leqslant(2 K+1) \omega(f, \varepsilon)$. Таким образом, $\|f-\hat{g}\| \leqslant(2 K+1) \omega(f, \varepsilon)$. Отметим, что пространство $X$ удовлетворяет условиям теоремы 1. Без потери общности можно считать, что $\delta \stackrel{\text { def }}{=}$ $\omega(f, \varepsilon)>0$. В силу замечания 2 к теореме 1 найдется $2 D K \frac{\omega(f, \varepsilon)}{\varepsilon}$-липшицево локально $\alpha$-гладкое отображение, такое, что $\|\hat{g}-\varphi\| \leqslant \delta=\omega(f, \varepsilon)$. Тогда $\|f-\varphi\| \leqslant\|f-\hat{g}\|+\|\hat{g}-\varphi\| \leqslant 2(K+1) \omega(f, \varepsilon)$. Теорема доказана.

Отметим, что во время написания работы автору стал известен следующий результат Наора, Переса, Шрама и Шефилда: для любого подмножества $M \subset$ $X$, где $X$ - банахово пространство с модулем гладкости 2-го порядка (в частности, таким будет любое пространство $\left.X \in\left(\mathscr{H}^{\alpha}\right)\right)$, и произвольного банахова пространства $Y$ с модулем выпуклости 2-го порядка всякое липшицево отображение $f: M \rightarrow Y$ продолжается до липшицева отображения на всем пространстве $X$ (константа Липшица при этом увеличивается в некоторое постоянное число раз, причем это число зависит от $X, Y)$. Это позволяет получить утверждение, аналогичное теореме 2 , заменив $l_{2}$ на $L_{p}$ для $p \in(1,2]$.

ДокАзАТЕльство теоремы 3. В работе [9, теорема 1, с. 122] было доказано, что найдется абсолютная константа $K>0$, такая, что для любого $\varepsilon>0$ существует $K \frac{\omega(f, \varepsilon)}{\varepsilon}$-липшицева функция $\psi: l_{2} \rightarrow \mathbb{R}$, производная которой является $K \frac{\omega(f, \varepsilon)}{\varepsilon^{2}}$-липшицевым отображением и для которой $\|f-\psi\| \leqslant K \omega(f, \varepsilon)$. Без потери общности можно считать, что $\delta \stackrel{\text { def }}{=} \omega(f, \varepsilon)>0$. В силу замечания 3 найдется абсолютная константа $G>0$ и локально $\infty$-гладкая $D K \frac{\omega(f, \varepsilon)}{\varepsilon}$-липшицева функция $\varphi: l_{2} \rightarrow \mathbb{R}$, производная которой является $G K \frac{\omega(f, \varepsilon)}{\varepsilon^{2}}$-липшицевым отображением, такая, что $\|\psi-\varphi\| \leqslant \delta=\omega(f, \varepsilon)$. Отсюда вытекает, что $\|f-\varphi\| \leqslant \| f-$ $\psi\|+\| \psi-\varphi \| \leqslant(K+1) \omega(f, \varepsilon)$. И, следовательно, для $C=\max \{K+1, D K, G K\}$ и функции $\varphi$ получается требуемое утверждение. Теорема доказана.

\section{ЛитературА}

[1] Дж. Иллс, “Основания глобального анализа", УМН, 24:3 (147) (1969), 157-210.

[2] J. Kurzweil, "On approximation in real Banach spaces", Studia Math., 14:2 (1954), 214-231.

[3] F. A. Valentine, "A Lipschitz conditions preserving extension for a vector function", Amer. J. Math., 67:1 (1945), 83-93. 
[4] А. С. Немировский, Гладкая и полиномиальная аппроксимация непрерывных бункций на гилъбертовом пространстве, Дисс. канд. физ.-мат. наук, МГУ, мехмат, 1973.

[5] С. М. Семенов, "О симметрических функциях класса $D_{u}^{2}(H)$ ", Функи. анализ и его прил., 6:3 (1972), 85-86.

[6] С. М. Семенов, Симметрические функции на пространствах $L_{p}$, Дисс. канд. физ.-мат. наук, МГУ, мех-мат, 1973.

[7] А. С. Немировский, С. М. Семенов, "О полиномиальной аппроксимации на гильбертовом пространстве", Матем. сб., 92:2 (1973), 257-281.

[8] И. Г. Царьков, "Сглаживание равномерно непрерывных отображений в пространствах $L_{p}$ ", Матем. заметки, 54:3 (1993), 123-140.

[9] И. Г. Царьков, “Сглаживание абстрактных функций”, Матем. сб., 185:11 (1994), 119-144.

[10] И. Г. Царьков, “Линейные методы в некоторых задачах сглаживания”, Maтем. заметки, 56:6 (1994), 64-87.

[11] И. Г. Царьков, "Приближение векторнозначных функций многочленами”, Функи. анализ и его прил., 29:3 (1995), 93-95.

[12] И. Г. Царьков, “О продолжении и сглаживании векторнозначных функций”, Изв. PAH, 59:4 (1995), 187-220.

[13] И. Г. Царьков, "Некоторые вопросы продолжения”, Матем. заметки, 58:6 (1995), 906-916.

[14] И. Г. Царьков, “О гладких выборках из множеств почти чёбышевских центров”, Вестник МГУ, сер. 1, матем., мех., 1996, № 2, 92-94.

[15] И. Г. Царьков, “Сглаживание функций на гильбертовом шаре”, Трудъ $М И Р A H$, 219 (1997), 410-419.

[16] И. Г. Царьков, “Сглаживание функций в конечномерных пространствах", Изв. РАН, 61:1 (1997), 199-214.

[17] И. Г. Царьков, "Продолжение гильбертовозначных липшицевых отображений”, Вестник МГУ, сер. 1, матем., мех., 1999, № 6, 9-16.

[18] Y. Benyamini, J. Lindenstrauss, Geometric Nonlinear Functional Analysis, vol. 1, Colloquium Publications, vol. 48, Amer. Math. Soc., Providence, RI, 2000.

[19] J. Lindenstrauss, L. Tzafriri, Classical Banach Spaces, I, II, Springer-Verlag, Berlin, 1996.

[20] I. Singer, Bases in Banach Spaces, I, Springer-Verlag, 1970.

Московский государственный университет

им. М. В. Ломоносова,

механико-математический факультет

e-mail: tsar@mech.math.msu.su

Поступило в редакцию 30 ноября 2004 г. 